\title{
Microwave-assisted solvent free efficient synthesis of 1,3,4-oxadiazole-2(3H)- thiones and their potent in vitro urease inhibition activity
}

\author{
Sohail Anjum Shahzada,*, Muhammad Yarb, Zulfiqar Ali Khanc, Islam Ullah Khand, \\ Syed Ali Raza Naqvic, Nasir Mahmoode and Khalid Mohammed Khan ${ }^{\mathrm{f}}$ \\ a Department of Chemistry, COMSATS Institute of Information Technology, Abbottabad, 22060, Pakistan \\ ${ }^{\mathrm{b}}$ Interdisciplinary Research Center in Biomedical Materials, COMSATS Institute of Information Technology, Lahore, 54000, Pakistan \\ c Department of Chemistry, Government College University, Faisalabad, 38000, Pakistan \\ d Department of Chemistry, Government College University, Lahore, 54000, Pakistan \\ e Department of Allied Sciences and Chemical Pathology, University of Health Sciences, Lahore, 54600, Pakistan \\ ${ }^{\mathrm{f}}$ Hussain Ibrahim Jamal Research Institute of Chemistry, International Center for Chemical and Biological Sciences, University of Karachi, Karachi, 75270, Pakistan \\ ${ }^{*}$ Corresponding author at: Department of Chemistry, COMSATS Institute of Information Technology, Abbottabad, 22060, Pakistan. \\ Tel.: +92.992.383591-5; fax: +92.992.383441. E-mail address: sohail_chem@yahoo.com (S. A. Shahzad).
}

\section{ARTICLE INFORMATION}

Received: 18 November 2011

Received in revised form: 10 January 2012

Accepted: 03 February 2012

Online: 30 June 2012

\section{KEYWORDS}

\section{High yield}

Hydrazides

Solvent free

Urease inhibition

Microwave-irradiation

Substituted-1,3,4-oxadiazole

\section{Introduction}

The discovery of novel urease inhibitors has attracted the attention of many research groups [1-5] due to their wide applications against urea amidohydrolase, which is a nickel dependent enzyme that is responsible for the bacteria growth both in the soil and in the human body. Urease inhibition is not only responsible for maintaining human and animals good health but also has wide applications in agriculture [6]. Bacterial ureases are large heteropolymeric metalloproteins with nickel(II) ions present in their active sites [7-12].

Urea amidohydrolase, EC 3.5.1.5 is widely found in animals and plants kingdom. Many microorganisms use this enzyme in a reaction, which provides nitrogen for growth [13], and the enzyme also plays an important role in plant nitrogen metabolism during the germination process $[14,15]$. The presence of urease activity in soil is exploited in the widespread agricultural practice of urea-based fertilizer application for enhancing crop yields. Unfortunately, excessive levels of soil urease can degrade fertilizer urea too rapidly and result in phytopathic effects and loss of volatilized ammonia [16]. The urease is a virulence factor in certain human and animal pathogens; it participates in the development of kidney stones, pyelonephritis, peptic ulcers, and other disease states [17-20]. The obvious remedy for treating bacterial infection with antimicrobials, however, has often proven futile [21], and only a few combination regiments has reached clinical practice. Thus the need for alternative or novel treatment to inhibit urease activity for the possible development of highly needed therapy for urease mediated bacterial infections is evident.
Heterocycles especially 1,3,4-oxadiazole are some of the most important motifs in medicinally important compounds. The substituted oxadiazoles serve both as biomimetic and reactive pharmacophores. Such compounds offer a privileged motif in medicinal chemistry; such as antidiabetic [22] anticancer [23], antialzimer activity, inhibitors of glycogen synthase kinase-3 $\beta$ [24], insecticidal [25], bactericidal [26], hypoglycemic [27], analgesic, anticonvulsive, antiemetic, diuretic [28], muscle relaxant $[29,30]$, herbicidal $[31,32]$ and fungicidal activity $[33,34]$.

Since the discovery of potent urease inhibitors is an important area of pharmaceutical research, therefore several classes of compounds have been tested and found to exhibit significant inhibitory activities against urease enzyme [35-38]. Similarly, 5-membered heterocycles such as 1,3,4-oxadiazoles and 1,2,4-triazoles are also known to inhibit the bacterial ureases [39-41]. Previously we have tested urease inhibitors activities of some of 1,3,4-oxadiazoles [41], encouraged by these results and in search of more potent new urease inhibitors, herein we propose microwave assisted synthesis of oxadiazoles bearing different level of substituents at C- 5 .

\section{Experimental}

\subsection{Instrumentation}

Melting points were determined on a Büchi 434 melting point apparatus and are uncorrected. NMR was performed on Bruker AM 300, 400 and $500 \mathrm{MHz}$. CHN analysis was performed on a Carlo Erba Strumentazion-Mod-1106 Italy. 
Infrared Spectra (IR) was recorded on JASCO IR-A-302 spectrometer. Electron Impact Mass Spectra (EI-MS) were recorded on a Finnigan MAT-31A Germany. Thin Layer chromatography (TLC) was performed on pre-coated silica gel glass plates (Kieselgel 60, 254, E. Merck, Germany). Chromatograms were visualized by UV at 254 and $365 \mathrm{~nm}$.

\subsection{Urease assay and inhibition}

Reaction mixtures comprising $25 \mu \mathrm{L}$ (1.0 mM concentration) of enzyme (Jack bean Urease) solution and $55 \mu \mathrm{L}$ of buffers containing $100 \mathrm{mM}$ urea were incubated with $5 \mu \mathrm{L}$ of test compounds ( $1.0 \mathrm{mM}$ concentration) at $30{ }^{\circ} \mathrm{C}$ for $15 \mathrm{~min}$ in $96-$ well plates. Urease activity was determined by measuring ammonia production using the indophenol method as described by Weatherburn [42]. Briefly, $45 \mu \mathrm{L}$ each of phenol reagent $(1 \% \quad(\mathrm{w} / \mathrm{v})$ phenol and $0.005 \% \quad(\mathrm{w} / \mathrm{v})$ sodium nitroprusside) and $70 \mu \mathrm{L}$ of alkali reagent $(0.5 \%(\mathrm{w} / \mathrm{v}) \mathrm{NaOH}$ and $0.1 \%$ active chlorine, $\mathrm{NaOCl}$ ) were added to each well. The increasing absorbance at $630 \mathrm{~nm}$ was measured after $50 \mathrm{~min}$, using a microplate reader (Molecular Device, USA). All reactions were performed in triplicate in a final volume of 200 $\mu \mathrm{L}$. The results (change in absorbance per min.) were processed by using SoftMax Pro software (Molecular Device, USA). All the assays were performed at $\mathrm{pH}=8.2(0.01 \mathrm{M}$ $\mathrm{K}_{2} \mathrm{HPO}_{4} 3 \mathrm{H}_{2} \mathrm{O}, 1.0 \mathrm{mM}$ EDTA and $0.01 \mathrm{M} \mathrm{LiCl}_{2}$ ). Percentage inhibitions were calculated from the formula 100$\left(\mathrm{OD}_{\text {testwell }} / \mathrm{OD}_{\text {control }}\right) \times 100$. Thiourea was used as the standard inhibitor of urease.

\subsection{Synthesis of compounds 2(a-r) [43]}

A mixture of respective hydrazide $(10 \mathrm{mmol})$, potassium hydroxide $(0.56 \mathrm{~g}, 10 \mathrm{mmol})$ and alumina were finely ground in a glove box with a mortar and pestle. Then carbon disulfide $(1.2$ $\mathrm{mL}, 20 \mathrm{mmol}$ ) was added to this mixture in a pyrex glass vial, which was placed in a screw-capped thick-walled Teflon ${ }^{\circledR}$ vessel. Microwave-irradiation (MW domestic type oven $900 \mathrm{~W}$ with a frequency $2450 \mathrm{MHz}$, Dawlance, Pakistan) was applied for 3-7 min. After the completion of reaction (TLC analysis), ethanol was added into reaction mixture and filtered. Filtrate was evaporated; distilled water was added to semi-solid material and acidified with hydrochloric acid to $\mathrm{pH}=4$. Precipitates so obtained were filtered and dried to afford off white solid compound $\mathbf{2 a - r}$ and then recrystallized from ethanol:water (50:50) mixture (Table 1).

5(2'-Hydroxyphenyl-1,3,4-oxadiazole-2(3H)-thione (2a): Yield: 1.33 g (89\%). M.p.: 200-201 ${ }^{\circ} \mathrm{C} . \mathrm{R}_{f}=0.67$ (Ethyl acetate: hexane $=1: 1)$. FT-IR $\left(\mathrm{KBr}, v_{\max }, \mathrm{cm}^{-1}\right): 3364(\mathrm{NH}), 1618$ $(\mathrm{C}=\mathrm{N}), 1309(\mathrm{C}=\mathrm{S}), 1051(\mathrm{C}-\mathrm{O}-\mathrm{C})$. MS $(\mathrm{m} / \mathrm{z}, \%): 194\left(\mathrm{M}^{+}, 100\right)$, 134 (1), 121 (99), 119 (16), 93 (7), 65 (8). ${ }^{1} \mathrm{H}$ NMR (400 MHz, Acetone- $\left.d_{6}, \delta, \mathrm{ppm}\right): 14.33$ (bs, $\left.1 \mathrm{H}, \mathrm{NH}\right), 8.84(\mathrm{br} \mathrm{s}, 1 \mathrm{H}, \mathrm{OH}$ ), $7.61\left(\mathrm{~m}, 1 \mathrm{H}, \mathrm{H}-4^{\prime}\right), 7.08\left(\mathrm{~d}, 1 \mathrm{H}, \mathrm{H}-3^{\prime}\right), 7.07(\mathrm{dd}, 1 \mathrm{H}, J=8.1 \mathrm{~Hz}, J=$ $\left.6.4 \mathrm{~Hz}, \mathrm{H}-5^{\prime}\right), 7.01\left(1 \mathrm{H}, J=7.7 \mathrm{~Hz}, J=5.0, J=1.5 \mathrm{~Hz}, \mathrm{H}-6^{\prime}\right)$. Anal. calcd. for $\mathrm{C}_{8} \mathrm{H}_{6} \mathrm{~N}_{2} \mathrm{O}_{2} \mathrm{~S}$ : C, 49.47; $\mathrm{H}, 3.11 ; \mathrm{N}, 14.42$. Found: $\mathrm{C}$, 49.49; H, 3.15; N, 14.46\%.

5(4'-Methoxyphenyl)-1,3,4-oxadiazole-2(3H)-thione (2b): Yield: 1.41 g (94\%). M.p.: 190-191 ${ }^{\circ} \mathrm{C} . \mathrm{R}_{f}=0.65$ (Ethyl acetate: hexane $=1: 1)$. FT-IR $\left(\mathrm{KBr}, v_{\max }, \mathrm{cm}^{-1}\right): 3399(\mathrm{NH}), 1659$ $(\mathrm{C}=\mathrm{N}), 1333(\mathrm{C}=\mathrm{S}), 1019(\mathrm{C}-\mathrm{0})$. MS ( $\mathrm{m} / \mathrm{z}, \%): 208\left(\mathrm{M}^{+}, 100\right)$, 148 (54), 135 (12), 133 (88), 107 (3), 105 (20), 92 (10), 77 (13), 64 (14), 51 (18). ${ }^{1} \mathrm{H}$ NMR (500 MHz, $\left.\mathrm{CD}_{3} \mathrm{OD}, \delta, \mathrm{ppm}\right): 14.23$ (br s, $1 \mathrm{H}, N H), 7.84\left(\mathrm{~d}, 2 \mathrm{H}, J=8.8 \mathrm{~Hz}, \mathrm{H}-2^{\prime} / 6^{\prime}\right), 7.05(\mathrm{~d}, 2 \mathrm{H}, J=8.8$ $\left.\mathrm{Hz}, \mathrm{H}-3^{\prime} / 5^{\prime}\right), 3.61\left(\mathrm{~s}, 3 \mathrm{H}, \mathrm{OCH}_{3}\right.$ ). Anal. calcd. for $\mathrm{C}_{9} \mathrm{H}_{8} \mathrm{~N}_{2} \mathrm{O}_{2} \mathrm{~S}: \mathrm{C}$, 51.91; H, 3.87; N, 13.45. Found: C, 51.96; H, 3.83; N, 13.49\%.

5(3'-Nitrophenyl)-1,3,4-oxadiazole-2(3H)-thione (2c): Yield: $1.38 \mathrm{~g}(92 \%)$. M.p.: $142-144^{\circ} \mathrm{C} . \mathrm{R}_{f}=0.66$ (Ethyl acetate:hexane $=1: 1)$. FT-IR (KBr, $\left.v_{\max }, \mathrm{cm}^{-1}\right): 3321(\mathrm{NH}), 1639(\mathrm{C}=\mathrm{N}), 1318$ (C=S), 1078 (C-O-C). MS $(\mathrm{m} / \mathrm{z}, \%): 223\left(\mathrm{M}^{+}, 81\right), 163(100), 150$ (5), 133 (119), 117 (30), 105 (5), 104 (17), 102 (13), 76 (39). ${ }^{1} \mathrm{H}$ NMR (300 MHz, DMSO, $\left.\delta, \mathrm{ppm}\right): 14.61$ (s, NH), 8.50 (bs, 1H,
H-2'), 8.44 (br. d, $1 \mathrm{H}, J=8.3 \mathrm{~Hz}, \mathrm{H}-6^{\prime}$ ), 8.28 (br. d, $1 \mathrm{H}, J=7.8 \mathrm{~Hz}$, $\mathrm{H}-4^{\prime}$ ), $8.01\left(\mathrm{t}, 1 \mathrm{H}, J=8.0 \mathrm{~Hz}, \mathrm{H}-5^{\prime}\right)$. Anal. calcd. for $\mathrm{C}_{8} \mathrm{H}_{5} \mathrm{~N}_{3} \mathrm{O}_{3} \mathrm{~S}: \mathrm{C}$, 43.05; H, 2.26; N, 18.83. Found: C, 43.08; H, 2.29; N, 18.80\%.

5(4'-Nitrophenyl)-1,3,4-oxadiazole-2(3H)-thione (2d): Yield: $1.39 \mathrm{~g}$ (93\%). M.p.: $>250{ }^{\circ} \mathrm{C}$ (Decompose). $\mathrm{R}_{f}=0.64$ (Ethyl acetate:hexane $=1: 1)$. FT-IR $\left(\mathrm{KBr}, v_{\max }, \mathrm{cm}^{-1}\right): 3366(\mathrm{NH}), 1632$ $(\mathrm{C}=\mathrm{N}), 1328(\mathrm{C}=\mathrm{S}), 1086(\mathrm{C}-\mathrm{O}-\mathrm{C})$. MS $(\mathrm{m} / \mathrm{z}, \%): 223\left(\mathrm{M}^{+}, 79\right)$, 163 (100), 150 (4), 133 (21), 117 (28), 105 (4), 104 (12), 102 (9), 76 (34). ${ }^{1} \mathrm{H}$ NMR $\left(400 \mathrm{MHz}, \mathrm{CD}_{3} \mathrm{OD}, \delta, \mathrm{ppm}\right): 14.73$ (bs, $1 \mathrm{H}$, $\mathrm{NH}), 8.40$ (d, $\left.2 \mathrm{H}, J=8.7 \mathrm{~Hz}, \mathrm{H}-3^{\prime}, 5^{\prime}\right), 8.17$ (d, $2 \mathrm{H}, J=8.7 \mathrm{~Hz}, \mathrm{H}-$ $2^{\prime}, 6^{\prime}$ ). Anal. calcd. for $\mathrm{C}_{8} \mathrm{H}_{5} \mathrm{~N}_{3} \mathrm{O}_{3} \mathrm{~S}$ : C, 43.05; $\mathrm{H}, 2.26$; N 18.83 . Found: C, 43.01; H, 2.22; N, 18.86\%.

$5\left(3^{\prime}, 4^{\prime}, 5^{\prime}\right.$-Trimethoxyphenyl)-1,3,4-oxadiazole-2(3H)-thione (2e): Yield: $1.36 \mathrm{~g}(91 \%)$. M.p.: $175-176{ }^{\circ} \mathrm{C} . \mathrm{R}_{f}=0.59$ (Ethyl acetate:hexane $=1: 1)$. FT-IR $\left(\mathrm{KBr}, v_{\max }, \mathrm{cm}^{-1}\right): 3171(\mathrm{NH}), 1579$ $(\mathrm{C}=\mathrm{N}), 1331(\mathrm{C}=\mathrm{S}), 1041$ (C-O-C). MS ( $/ \mathrm{m}, \mathrm{z}, \%): 268$ (100), 208 (32), 193 (70), 178 (12), 167 (6), 152 (7), 135 (13). ${ }^{1 \mathrm{H}}$ NMR (300 MHz, DMSO- $d_{6}, \delta, \mathrm{ppm}$ ): 14.81 (br s, $\left.1 \mathrm{H}, N H\right), 7.09$ (s, 2H, $\left.\mathrm{H}-2^{\prime}, 6^{\prime}\right), 3.84\left(\mathrm{~s}, 6 \mathrm{H}, \mathrm{OCH}_{3}-3^{\prime} / 5^{\prime}\right), 3.72\left(\mathrm{~s}, 3 \mathrm{H}, \mathrm{OCH}_{3}-4^{\prime}\right)$. Anal. calcd. for $\mathrm{C}_{11} \mathrm{H}_{12} \mathrm{~N}_{2} \mathrm{O}_{4} \mathrm{~S}$ : C, 49.24; $\mathrm{H}, 4.51$; N, 10.44. Found: C, 49.28; $\mathrm{H}, 4.56 ; \mathrm{N}, 10.47 \%$.

5(2'-Chlorophenyl-1,3,4-oxadiazole-2(3H)-thione (2f): Yield: $1.47 \mathrm{~g}(98 \%)$ ). M.p.: $157-158{ }^{\circ} \mathrm{C} . \mathrm{R}_{f}=0.69$ (Ethyl acetate:hexane $=1: 1)$. FT-IR $\left(\mathrm{KBr}, v_{\max }, \mathrm{cm}^{-1}\right): 1051(\mathrm{C}-\mathrm{O}-\mathrm{C}), 1614(\mathrm{C}=\mathrm{N}), 3583$ $(\mathrm{NH})$. MS $(\mathrm{m} / \mathrm{z}, \%): 214\left(\mathrm{M}^{+}, 17\right), 212\left(\mathrm{M}^{+}, 47\right), 179(3), 154$ (32), 152 (100), 141 (4), 139 (11), 137 (14), 117 (10), 113 (6), 111 (19), 102 (15), 76 (9). ${ }^{1} \mathrm{H}$ NMR ( $\left.500 \mathrm{MHz}, \mathrm{CD}_{3} \mathrm{OD}, \delta, \mathrm{ppm}\right)$ : 14.63 (bs, 1H, NH), 7.89 (dd, 1H, $J=7.0,1.5 \mathrm{~Hz}, \mathrm{H}-6^{\prime}$ ), 7.61 (dd, $1 \mathrm{H}, J=7.2,1.7 \mathrm{~Hz}, \mathrm{H}-3^{\prime}$ ), 7.56 (dd, $1 \mathrm{H}, J=7.1 \mathrm{~Hz}, 1.9 \mathrm{~Hz}, \mathrm{H}-4^{\prime}$ ), $7.48\left(\mathrm{t}, 1 \mathrm{H}, J=7.1 \mathrm{~Hz}, \mathrm{H}-5^{\prime}\right)$. Anal. calcd. for $\mathrm{C}_{8} \mathrm{H}_{5} \mathrm{ClN}_{2} \mathrm{OS}$ : C, 45.18; H, 2.37; N, 13.17. Found: C, 45.23; H, 2.41; N, 13.16\%.

5(3'-Chlorophenyl-1,3,4-oxadiazole-2(3H)-thione $(\mathbf{2 g})$ : Yield: $1.42 \mathrm{~g}(95 \%)$. M.p.: $178-179{ }^{\circ} \mathrm{C} . \mathrm{R}_{f}=0.69$ (Ethyl acetate:hexane $=1: 1)$. FT-IR (KBr, $\left.v_{\max }, \mathrm{cm}^{-1}\right): 1063(\mathrm{C}-\mathrm{O}-\mathrm{C}), 1608(\mathrm{C}=\mathrm{N}), 3594$ (NH). MS $(\mathrm{m} / \mathrm{z}, \%): 214\left(\mathrm{M}^{+}, 48\right), 212\left(\mathrm{M}^{+}, 100\right), 179(3), 155$ (6), 154 (37), 153 (11), 152 (100), 141 (5), 139 (17), 137 (12), 117 (7), 102 (8), 76 (7). ${ }^{1}$ H-NMR (500 MHz, DMSO-d 6 ): 14.49 (bs, $1 \mathrm{H}, N H$ ), 7.85 (d, $\left.1 \mathrm{H}, J=1.6 \mathrm{~Hz}, \mathrm{H}-6^{\prime}\right), 7.83$ (d, $J=7.8 \mathrm{~Hz}, \mathrm{H}-$ $2^{\prime}$ ), 7.70 (dd, $1 \mathrm{H}, J=7.9 \mathrm{~Hz}, J=1.2 \mathrm{~Hz}, \mathrm{H}-4$ ), $7.60(\mathrm{t}, 1 \mathrm{H}, J=7.9$ $\mathrm{Hz}, J=7.9 \mathrm{~Hz}, \mathrm{H}-3^{\prime}$ ). Anal. calcd. for $\mathrm{C}_{8} \mathrm{H}_{5} \mathrm{ClN}_{2} \mathrm{OS}: \mathrm{C}, 45.18 ; \mathrm{H}$, 2.37; N, 13.17. Found: C, 45.23; H, 2.42; N, $13.15 \%$.

5(4'-Chlorophenyl-1,3,4-oxadiazole-2(3H)-thione $\mathbf{2} \mathbf{2 h})$ : Yield: $1.38 \mathrm{~g}(92 \%)$. M.p.: $173-174{ }^{\circ} \mathrm{C} . \mathrm{R}_{f}=0.69$ (Ethyl acetate:hexane $=1: 1)$. FT-IR (KBr, $\left.v_{\max }, \mathrm{cm}^{-1}\right): 1023$ (C-O-C), $1669(\mathrm{C}=\mathrm{N}), 3348$ $(\mathrm{NH})$. MS (m/z, \%): $214\left(\mathrm{M}^{+}, 35\right), 212\left(\mathrm{M}^{+}, 100\right), 179(3), 154$ (30), 152 (82), 141 (4), 139 (14), 137 (14), 117 (5), 102 (8), 76 (7). ${ }^{1} \mathrm{H}$ NMR (500 MHz, DMSO- $\left.d_{6}, \delta, \mathrm{ppm}\right): 14.52(\mathrm{bs}, 1 \mathrm{H}, N H)$, 7.88 (d, $\left.2 \mathrm{H}, J=8.6 \mathrm{~Hz}, \mathrm{H}-2^{\prime} / 6^{\prime}\right), 7.65$ (d, $2 \mathrm{H}, J=8.6 \mathrm{~Hz}, \mathrm{H}-3^{\prime} / 5^{\prime}$ ). Anal. calcd. for $\mathrm{C}_{8} \mathrm{H}_{5} \mathrm{ClN}_{2} \mathrm{OS}$ : C, 45.18; $\mathrm{H}, 2.37 ; \mathrm{N}, 13.17$. Found: C, 45.15; H, 2.39; N, 13.14\%.

5(4'-Flourophenyl-1,3,4-oxadiazole-2(3H)-thione (2i): Yield: $1.44 \mathrm{~g}(96 \%)$. M.p.: $192-193{ }^{\circ} \mathrm{C} . \mathrm{R}_{f}=0.68$ (Ethyl acetate:hexane $=1: 1)$. FT-IR (KBr, $\left.v_{\max }, \mathrm{cm}^{-1}\right): 3431(\mathrm{NH}), 1641(\mathrm{C}=\mathrm{N}), 1328$ (C=S), 1020 (C-O). MS (m/z, \%): $196\left(\mathrm{M}^{+} 100\right), 163$ (10), 137 (12), 136 (100), 12 (22), 121 (38), 95 (33), 69 (5). ${ }^{1} \mathrm{H}$ NMR (300 MHz, DMSO- $\left.d_{6}, \delta, p p m\right): 14.71$ (bs, $\left.1 \mathrm{H}, \mathrm{NH}\right), 7.89-7.95(\mathrm{~m}, 2 \mathrm{H}$, $\left.\mathrm{H}-3^{\prime}, 5^{\prime}\right)$, 7.37-7.45 (m, $\left.2 \mathrm{H}, \mathrm{H}-2^{\prime}, 6^{\prime}\right)$. Anal. calcd. for $\mathrm{C}_{8} \mathrm{H}_{5} \mathrm{FN}_{2} \mathrm{OS}$ : C, 48.97; H, 2.57; N, 14.28. Found: C, 48.92; H, 2.53; N, 14.29\%.

5(2'-Bromophenyl-1,3,4-oxadiazole-2(3H)-thione (2j): Yield: $1.18 \mathrm{~g}(79 \%)$. M.p.: $190-191{ }^{\circ} \mathrm{C} . \mathrm{R}_{f}=0.69$ (Ethyl acetate:hexane $=1: 1)$. FT-IR (KBr, $\left.v_{\max } \mathrm{cm}^{-1}\right): 1063(\mathrm{C}-\mathrm{O}-\mathrm{C}), 1649(\mathrm{C}=\mathrm{N}), 3219$ (NH). MS (m/z, \%): $258\left(\mathrm{M}^{+}, 100\right), 256\left(\mathrm{M}^{+}, 99\right), 198(92), 196$ (93), 185 (64), 183 (78), 181 (13), 157 (21), 155 (24), 76 (19), 74 (7), 50 (13). ${ }^{1} \mathrm{H}$ NMR (500 MHz, $\left.\mathrm{CD}_{3} \mathrm{OD}, \delta, \mathrm{ppm}\right): 14.53$ (bs, $1 \mathrm{H}, \mathrm{NH}), 7.82\left(\mathrm{dd}, 1 \mathrm{H}, J=7.7 \mathrm{~Hz}, 1.6 \mathrm{~Hz}, \mathrm{H}-6^{\prime}\right), 7.78(\mathrm{~d}, 1 \mathrm{H}, J=$ $7.5 \mathrm{~Hz}, \mathrm{H}-3^{\prime}$ ), 7.52 (t, $1 \mathrm{H}, J=7.5 \mathrm{~Hz}, J=7.5 \mathrm{~Hz}, \mathrm{H}-4^{\prime}$ ), 7.47 (ddd, $\left.1 \mathrm{H}, J=7.5 \mathrm{~Hz}, J=1.6 \mathrm{~Hz}, \mathrm{H}-5^{\prime}\right)$. Anal. calcd. for $\mathrm{C}_{8} \mathrm{H}_{5} \mathrm{BrN}_{2} \mathrm{OS}: \mathrm{C}$, 34.37; H, 1.96; N, 10.90. Found: C, 34.33; H, 1.98; N, 10.93\%.

5(3'-Bromophenyl-1,3,4-oxadiazole-2(3H)-thione (2k): Yield: 1.32 g (88\%). M.p.: 208-209 ${ }^{\circ}$ C. $\mathrm{R}_{f}=0.67$ (Ethyl acetate: hexane $=1: 1$ ). 
Table 1. Synthesis of 5-substituted-1,3,4-oxadiazole-2(3H)-thiones and tabular representation of urease inhibitory activity of compounds 2a-2r against standard inhibitor.

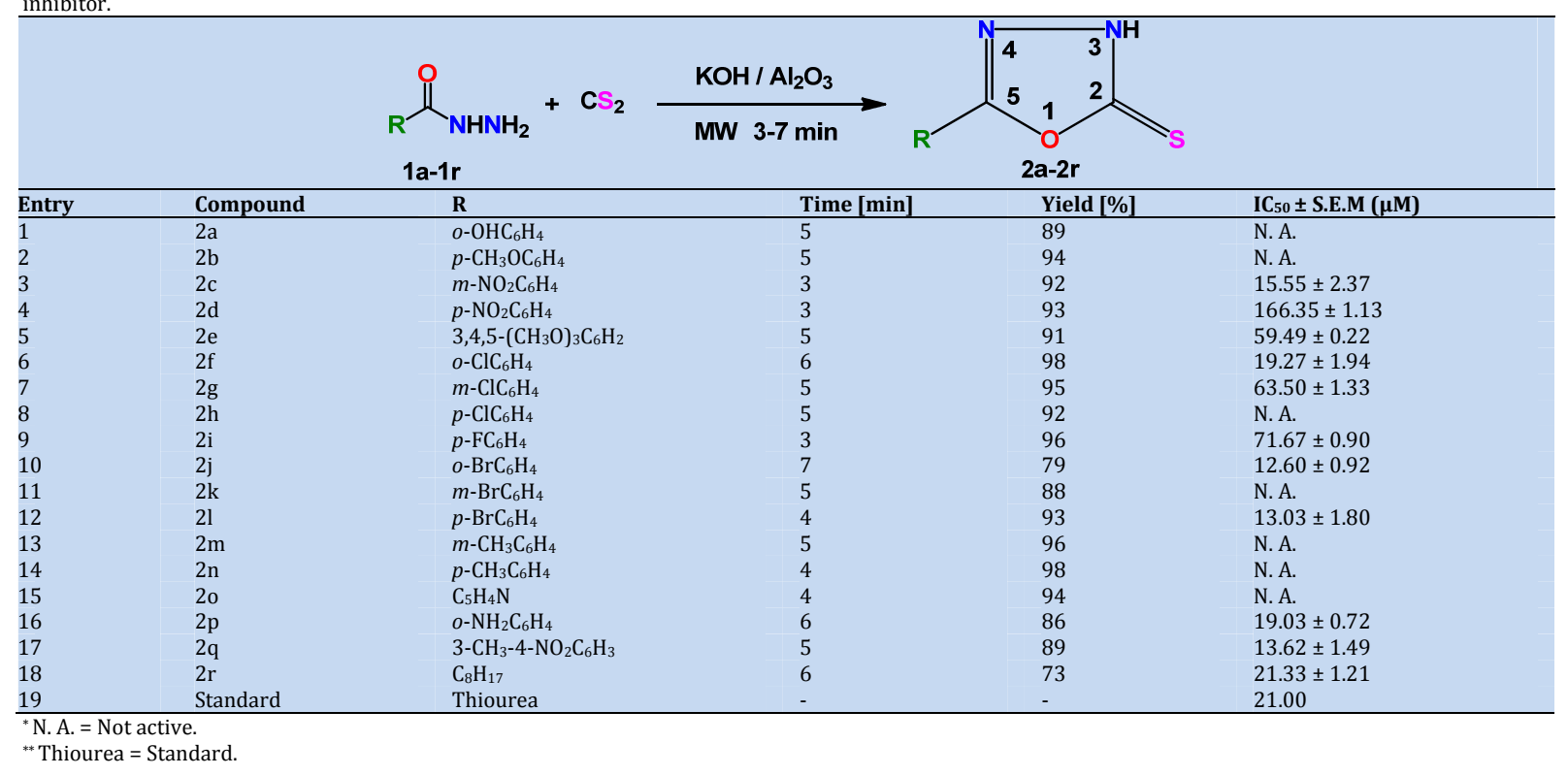

FT-IR (KBr, v $\left.v_{\max }, \mathrm{cm}^{-1}\right): 1069$ (C-O-C), 1631 (C=N), 3181 (NH). EI-MS (m/z, \%): $258\left(\mathrm{M}^{+}, 94\right), 256\left(\mathrm{M}^{+}, 100\right), 198(86)$, 197 (9), 196 (96), 185 (12), 183 (17), 157 (16), 155 (14), 117 (24), 102 (14), 76 (15). ${ }^{1} \mathrm{H}$ NMR (500 MHz, $\left.\mathrm{CD}_{3} \mathrm{OD}, \delta, \mathrm{ppm}\right)$ : 14.64 (bs, 1H, NH), 8.03 (d, 1H, $J=1.5 \mathrm{~Hz}, \mathrm{H}-2^{\prime}$ ), 7.87 (d, 1H, $J=$ $8.0 \mathrm{~Hz}, \mathrm{H}-6^{\prime}$ ), $7.74\left(\mathrm{dd}, 1 \mathrm{H}, J=8.0 \mathrm{~Hz}, J=1.5 \mathrm{~Hz}, \mathrm{H}-4^{\prime}\right), 7.46$ (t, $1 \mathrm{H}, J=8.0 \mathrm{~Hz}, \mathrm{H}-5^{\prime}$ ). Anal. calcd. for $\mathrm{C}_{8} \mathrm{H}_{5} \mathrm{BrN}_{2} \mathrm{OS}$ : C, 34.37; $\mathrm{H}$, 1.96; N 10.90. Found: C, 34.35; H, 1.98; N, 10.93\%.

5(4'-Bromophenyl-1,3,4-oxadiazole-2(3H)-thione (2l): Yield: $1.39 \mathrm{~g}(93 \%)$. M.p.: $230-231^{\circ} \mathrm{C} . \mathrm{R}_{f}=0.66$ (Ethyl acetate:hexane = 1:2). FT-IR (KBr, $\left.v_{\max }, \mathrm{cm}^{-1}\right): 1073(\mathrm{C}-\mathrm{O}-\mathrm{C}), 1633(\mathrm{C}=\mathrm{N}), 3280$ (NH). MS (m/z, \%): $258\left(\mathrm{M}^{+}, 60\right), 256\left(\mathrm{M}^{+}, 59\right), 198(47), 196$ (46), 185 (9), 184 (69), 183 (15), 181 (6), 157 (7), 155 (8), 117 (6), 76 (7), 50 (8). ${ }^{1} \mathrm{H}$ NMR (500 MHz, $\left.\mathrm{CD}_{3} \mathrm{OD}, \delta, \mathrm{ppm}\right): 14.59$ (bs, $1 \mathrm{H}, \mathrm{NH}$ ), 7.8 (d, 2H, $J=8.4 \mathrm{~Hz}, \mathrm{H}-2^{\prime} / 6^{\prime}$ ), 7.7 (d, $2 \mathrm{H}, J=8.4$ $\mathrm{Hz}, \mathrm{H}-3^{\prime} / 5^{\prime}$ ). Anal. calcd. for $\mathrm{C}_{8} \mathrm{H}_{5} \mathrm{BrN}_{2} \mathrm{OS}$ : C, 34.37; $\mathrm{H}, 1.96 ; \mathrm{N}$, 10.90. Found: C, 34.32; H, 1.98; N, 10.96\%.

5(3'-Methylphenyl-1,3,4-oxadiazole-2(3H)-thione (2m): Yield: 1.44 g (96\%). M.p.: 148-149 ${ }^{\circ} \mathrm{C} . \mathrm{R}_{f}=0.69$ (Ethyl acetate: hexane $=1: 2)$. FT-IR $\left(\mathrm{KBr}, v_{\max }, \mathrm{cm}^{-1}\right): 3400(\mathrm{NH}), 1635$ $(\mathrm{C}=\mathrm{N}), 1319$ (C=S), 1022 (C-O). MS (m/z, \%): $192\left(\mathrm{M}^{+}, 49\right), 132$ (100), 116 (10), 104 (17), 91 (59), 77 (17), 65 (34), 63 (20), 51 (24). ${ }^{1} \mathrm{H}$ NMR (500 MHz, $\left.\mathrm{CD}_{3} \mathrm{OD}, \delta, \mathrm{ppm}\right): 14.45$ (bs, $1 \mathrm{H}, \mathrm{NH}$ ), 7.71 (s, 1H, H-2') 7.67 (d, 1H, J = 6.1 Hz, H-2'), 7.39 (bs, 2H, H$3^{\prime} / 4^{\prime}$ ), 2.4 (s, $\mathrm{CH}_{3}$ ). Anal. calcd. for $\mathrm{C}_{9} \mathrm{H}_{8} \mathrm{~N}_{2} \mathrm{OS}$ : C, 56.23; $\mathrm{H}, 4.19$; N, 14.57. Found: C, 56.28; H, 4.17; N, 14.62\%.

5(4'-Methylphenyl-1,3,4-oxadiazole-2(3H)-thione (2n): Yield: $1.47 \mathrm{~g}$ (98\%). M.p.: $159-160{ }^{\circ} \mathrm{C} . \mathrm{R}_{f}=0.69$ (Ethyl acetate:hexane $=1: 2)$. FT-IR $\left(\mathrm{KBr}, v_{\max }, \mathrm{cm}^{-1}\right): 3409(\mathrm{NH}), 1636$ $(\mathrm{C}=\mathrm{N}), 1333(\mathrm{C}=\mathrm{S}), 1016(\mathrm{C}-\mathrm{O})$. MS $(\mathrm{m} / \mathrm{z}, \%): 192\left(\mathrm{M}^{+}, 52\right), 132$ (100), 119 (20), 117 (10), 104 (12), 102 (2), 91 (45), 65 (16). ${ }^{1} \mathrm{H}$ NMR (400 MHz, CD $\left.30 D, \delta, p p m\right): 14.53(\mathrm{bs}, 1 \mathrm{H}, \mathrm{NH}), 7.74(\mathrm{~d}$, $2 \mathrm{H}, J=8.1 \mathrm{~Hz} \mathrm{H}-2^{\prime} / 6^{\prime}$ ), 7.30 (d, 2H, $J=8.1 \mathrm{~Hz}, \mathrm{H}-3^{\prime} / 5^{\prime}$ ), 2.38 (s, $3 \mathrm{H}, \mathrm{CH}_{3}$ ). Anal. calcd. for $\mathrm{C}_{9} \mathrm{H}_{8} \mathrm{~N}_{2} \mathrm{OS}$ : C, 56.23; $\mathrm{H}, 4.19 ; \mathrm{N}, 14.57$. Found: C, 56.28; H, 4.14; N, 14.52\%.

5(3'-pyridyl-1,3,4-oxadiazole-2(3H)-thione (2o): Yield: 1.41 g (94\%). M.p.: 220 ०C. $\mathrm{R}_{f}=0.31$ (Ethyl acetate:hexane = 2:1). FTIR $\left(\mathrm{KBr}, v_{\max }, \mathrm{cm}^{-1}\right): 3471(\mathrm{NH}), 1651(\mathrm{C}=\mathrm{N}), 1338(\mathrm{C}=\mathrm{S}), 1010$ (C-0). MS (m/z, \%): $179\left(\mathrm{M}^{+}, 94\right), 119$ (100), 106 (5), 104 (12), 92 (43), 78 (61), 66 (6), 65 (14), 51 (40). ${ }^{1} \mathrm{H}$ NMR (500 MHz, DMSO- $d_{6}, \delta, \mathrm{ppm}$ ): 14.51 (bs, $1 \mathrm{H}, N H$ ), 9.05 (bs, $1 \mathrm{H}, \mathrm{H}-2^{\prime}$ ), 8.80 $\left(\mathrm{d}, 1 \mathrm{H}, J=6 \mathrm{~Hz}, \mathrm{H}-6^{\prime}\right), 8.30\left(\mathrm{~d}, 1 \mathrm{H}, J=7.86, \mathrm{H}-4^{\prime},\right), 7.65(\mathrm{dd}, 1 \mathrm{H}, J=$
7.65, 7.65 Hz, ${\mathrm{H}-5^{\prime}}^{\prime}$ ). Anal. calcd. for $\mathrm{C}_{7} \mathrm{H}_{5} \mathrm{~N}_{3} \mathrm{OS}$ : C, 46.29; $\mathrm{H}, 2.81$; N, 23.45. Found: C, 46.33; H, 2.85; N, 23.41\%.

5-(2'-Amino phenyl)-1,3,4-oxadiazole-2(3H)-thione (2p): Yield: 1.31 g (86 \%). M.p.: 156-157 ${ }^{\circ} \mathrm{C} . \mathrm{R}_{f}=0.68$ (Ethyl acetate:hexane $=1: 1)$. FT-IR $\left(\mathrm{KBr}, v_{\max }, \mathrm{cm}^{-1}\right): 1054(\mathrm{C}-\mathrm{O}-\mathrm{C})$, $1616(\mathrm{C}=\mathrm{N}), 3585(\mathrm{NH})$. MS ( $\mathrm{m} / \mathrm{z}, \%): 193\left(\mathrm{M}^{+}, 17\right), 177$ (100), 133 (47), 120 (15), 118 (33), 92 (15), 76 (10). 1H NMR (500 MHz, DMSO-d6, $\delta$, ppm): 14.63 (bs, $1 \mathrm{H}, \mathrm{NH}$ ), 7.89 (dd, $1 \mathrm{H}, J=7.0$ Hz, 1.5 Hz, H-6'), 7.88 (bs, 2H, NH ), 7.61 (dd, 1H, J = 7.2 Hz, 1.7 Hz, H-3') 7.55 (dd , 1H, $J=7.0 \mathrm{~Hz}, 1.9 \mathrm{~Hz}, \mathrm{H}-4^{\prime}$ ), 7.48 (t, 1H, $J=$ $7.0 \mathrm{~Hz}, \mathrm{H}-5^{\prime}$ ). Anal. calcd. for $\mathrm{C}_{8} \mathrm{H}_{7} \mathrm{~N}_{3} \mathrm{OS}$ : C, 49.73; $\mathrm{H}, 3.65$; $\mathrm{N}$, 21.75. Found: C, 49.71; H, 3.63; N, 21.74\%.

5-(3'-Methyl,4'-nitro phenyl)-1,3,4-oxadiazole-2(3H)-thione (2q): Yield: 1.33 g (89\%). M.p.: 240-241 ${ }^{\circ} \mathrm{C} . \mathrm{R}_{f}=0.58$ (Ethyl acetate:hexane $=1: 1)$. FT-IR $\left(\mathrm{KBr}, v_{\max }, \mathrm{cm}^{-1}\right): 1100(\mathrm{C}-\mathrm{O}-\mathrm{C})$ $1627(\mathrm{C}=\mathrm{N}), 3395(\mathrm{NH})$. EI-MS ( $\mathrm{m} / \mathrm{z}, \%): 237\left(\mathrm{M}^{+}, 100\right),(207)$, 204 (23), 191 (14), 178 (100), 176 (43), 164 (47), 163 (12), 162 (14), 149 (16), 136 (7), 132 (69), 90 (40), 75 (18). ${ }^{1} \mathrm{H}$ NMR (300 MHz, DMSO-d6, $\delta, \mathrm{ppm}$ ): 14.78 (bs, $1 \mathrm{H}, \mathrm{NH}$ ), 7.76 (s, 1H, H$2^{\prime}$ ), $7.92\left(\mathrm{dd}, J=8.4 \mathrm{~Hz}, 1.6 \mathrm{~Hz}, \mathrm{H}-6^{\prime}\right.$ ), 8.14 (d, $1 \mathrm{H}, J=8.5 \mathrm{~Hz}, \mathrm{H}-$ $5^{\prime}$ ). Anal. calcd. for $\mathrm{C}_{8} \mathrm{H}_{6} \mathrm{~N}_{2} \mathrm{OS}$ : C, 45.56; $\mathrm{H}, 2.97 ; \mathrm{N}, 17.71$. Found: C, 45.60; H, 2.99; N, 17.67\%.

5-n-Octyl-1,3,4-oxadiazole-2(3H)-thione (2r): Yield: $1.09 \mathrm{~g}$ (73\%). M.p.: 55-56 ${ }^{\circ} \mathrm{C} . \mathrm{R}_{f}=0.59$ (Ethyl acetate:hexane = 1:1) FT-IR (KBr, $\left.v_{\max }, \mathrm{cm}^{-1}\right): 3384(\mathrm{NH}), 2955\left(\mathrm{CH}_{2}\right.$-aliphatic) 1666 $(\mathrm{C}=\mathrm{N}), 1315(\mathrm{C}=\mathrm{S}), 1089$ (C-O). MS ( $\mathrm{m} / \mathrm{z}, \%): 214\left(\mathrm{M}^{+}, 8\right), 181$ (10), 158 (3), 143 (4). ${ }^{1} \mathrm{H}$ NMR (500 MHz, DMSO- $d_{6}, \delta, \mathrm{ppm}$ ): 14.26 (bs, $1 \mathrm{H}, \mathrm{NH}), 2.70\left(\mathrm{t}, 2 \mathrm{H}, J=7.3 \mathrm{~Hz}, \mathrm{CH}_{2}-1\right), 1.62(\mathrm{q}, 2 \mathrm{H}$, , $)$ $\left.=7.3 \mathrm{~Hz}, \mathrm{CH}_{2}-2\right), 1.26\left(\mathrm{~m}, 10 \mathrm{H},\left(\mathrm{CH}_{2}\right)_{5}\right), 0.84(\mathrm{t}, 3 \mathrm{H}, J=6.5 \mathrm{~Hz}$, $\mathrm{CH}_{3}$ ). Anal. calcd. for $\mathrm{C}_{10} \mathrm{H}_{18} \mathrm{~N}_{2} \mathrm{OS}$ : C, 56.01; H, 8.46; N, 13.71 . Found: C, 56.08; H, 8.52; N, 13.69\%.

\section{Results and discussion}

The reaction of hydrazides with carbon disulfide, in the presence of potassium hydroxide, and loaded over alumina under microwave irradiation afforded 5-substituted-1,3,4oxadiazole-2(3H)-thiones in good to excellent yields (Table 1 ). Presence of electron withdrawing substituents enhances the conversion rate and reaction completed in short time. Conversion of aromatic hydrazides into 1,3,4-oxadiazoles is more convenient and afforded $89-98 \%$ yield but in the case of 
aliphatic hydrazide conversion is poor and relatively less yield is obtained $(73 \%)$.

\subsection{In vitro urease inhibition activity}

The bioactivity was assessed according to literature protocol and thiourea was used as a standard inhibitor having $\mathrm{IC}_{50}$ value $21 \mu \mathrm{M}$ (Table 1). All the synthesized compounds were tested against urease inhibitory effects, and activity ranges from $12.60 \pm 0.92 \mu \mathrm{M}$ to $166.35 \pm 1.13 \mu \mathrm{M}$. Seven compounds $2 \mathbf{c}, \mathbf{2 f}, \mathbf{2 j}, \mathbf{2 l}, \mathbf{2 p}, \mathbf{2 q}$ and $2 \mathbf{r}$ demonstrated excellent inhibitory activity in the range $12.60 \mu \mathrm{M}-21.33 \mu \mathrm{M}$, whereas remaining compounds exhibited good to moderate enzyme inhibitory activity (59.49-71.67 $\mu \mathrm{M})$. The inhibitory activities of compound $\mathbf{2 c}, \mathbf{2 d}$ and $\mathbf{2 q}$ are expected due to the presence of a nitro group which possibly coordinates with nickel (active site) of the enzyme. Among nitro substituted compounds, para-nitro group with meta-methyl group $\mathbf{2 q}$ displayed a remarkable progress in the urease inhibition $(13.62 \pm 1.49 \mu \mathrm{M})$. The presence of nitro group on para-position $\mathbf{2 d}$ also presented an excellent inhibitory activity $(15.55 \pm 2.37 \mu \mathrm{M})$ while para-nitro group containing compound 2c displayed poor inhibitory activity $(166.35 \pm 1.13 \mu \mathrm{M})$. It was observed that compound $\mathbf{2 f}$ containing ortho-chloro- substituted phenyl motif showed excellent inhibition $(19.27 \pm 1.94 \mu \mathrm{M})$, meta-chloro substituted compound $2 \mathrm{~g}$ presented significant inhibition $(63.50 \pm 1.33$ $\mu \mathrm{M}$ ) and whereas para-chloro- substituted compound $\mathbf{2 h}$ was found to be inactive. Generally, it was observed that compounds having para substituted phenyl motifs showed poor inhibition. Among bromo group substituted derivatives $\mathbf{2} \mathbf{j}-\mathbf{l}$, ortho-bromo substituted phenyl group in compound $2 \mathbf{j}$ displayed tremendous inhibition $(12.60 \pm 0.92 \mu \mathrm{M})$ while meta substituted bromo compound $\mathbf{2 k}$ is inactive. The presence of bromo group on para-position of phenyl motif $2 \mathbf{l}$ caused an outstanding urease inhibition $(13.03 \pm 1.80 \mu \mathrm{M})$.

From the structure activity relationship we found that declines in activity are rationalized that as the steric hinderance increases, the activity decreases, which may be responsible for less interaction of the molecules with the nickel of the enzyme.

\section{Conclusion}

In summary, we have efficiently extended our microwave assisted methodology to the synthesis of 1,3,4-oxadiazoles-2$(3 \mathrm{H})$-thiones in good to excellent yield. The compounds showed good to excellent urease inhibition activities. The compound $\mathbf{2 j}$ was found to be most potent having $\mathrm{IC}_{50} 12.60 \pm 0.92 \mu \mathrm{M}$.

\section{Acknowledgements}

The authors are thankful to The Organisation for the Prohibition of Chemical Weapons (OPCW), Netherland and Higher Education Commission (HEC) Pakistan (Project No. 1910 and under IPFP) for their financial supports. Authors are also grateful to Department of Allied Sciences and Chemical Pathology, University of Health Sciences, Lahore, 54600, Pakistan for carrying out bioactivities of our compounds..

\section{References}

[1]. Dixon, N. E.; Gazzola, C.; Watters, J. J.; Blakeley, R. L.; Zerner, B. J. Am. Chem. Soc. 1975, 97, 4130-4131.

[2]. Amtul, Z; Atta-ur-Rahman; Siddiqui, R. A.; Choudhary, M. I. Curr. Med. Chem. 2002, 9, 1323-1348.

[3]. Andrews, R. K.; Dexter, A.; Blakeley, R. L.; Zerner, B. J. Am. Chem. Soc. 1986, 108, 7124-7125.

[4]. Faraci, W. S.; Yang, B. V.; O'Rourke, D.; Spencer, R. W. Bioorg. Med. Chem. 1995, 3, 605-610.

[5]. Kot, M.; Zaborska, W.; Orlinska, K. J. Enzym. Inhib. Med. Chem. 2001, 16, 507-516.

[6]. Collier, J. L.; Brahamsha, B.; Palenik, B. Microbiology 1999, 145, 447459.
[7]. Krajewska, B. J. Mol. Catal. B: Enzym. 2009, 59, 9-21.

[8]. Karplus, P. A.; Pearson, M. A.; Hausinger, R. P. Acc. Chem. Res. 1997, 30, 330-337.

[9]. Dixon, N. E.; Gazzola, C.; Blakeley, R. L.; Zerner, B. J. Am. Chem. Soc. 1975, 97, 4131-4133.

[10]. Ermler, U.; Grabarse, W.; Shima, S.; Goubeaud, M.; Thauer, R. K. Curr. Opin. Struct. Biol. 1998, 8, 749-758.

[11]. Jabri, E.; Carr, M. B.; Hausinger, R. P.; Karplus, P. A. Science 1995, 268 998-1004.

[12]. Benini, S.; Rypniewski, W. R.; Wilson, K. S.; Ciurli, S.; Mangani, S. J. Biol. Inorg. Chem. 1998, 3, 268-273.

[13]. Witte, C. P.; Tiller, S. A.; Taylor, M. A.; Davies, H. V. Plant Physiol. 2002, 128, 1129-1136.

[14]. Mobley, H. L. T.; Hausinger, R. P. Microbiol. Rev. 1989, 53, 85-100.

[15]. Zonia, L. E.; Stebbins, N. E.; Polacco, J. C. Plant Physiol. 1995, 107, 1097-1103.

[16]. Mulvaney, R. L.; Bremner, J. M. Soil Biochemistry, Paul, E. A.; Ladd, J. N.; Eds., Marcel Dekker, Inc., New York, 1981, 153-196.

[17]. Mobley, H. L. T.; Island, M. D.; Hausinger, R. P. Microbiol. Rev. 1995, 59, 451-480.

[18]. Williamson, J. S. Curr. Pharm. Des. 2001, 7, 355-392.

[19]. Estiu G.; Merz, Jr. K. M. J. Am. Chem. Soc. 2004, 126, 6932-6944.

[20]. Burne, R. A.; Chen, Y. Y. M. Microbes Infect. 2000, 2, 533-542.

[21]. Bayerdorffer, E.; Ottenjhan, R. Scand. J. Gastroenterol. 1988, 23, 93100.

[22]. Shingalapur, R. V.; Hosamani, K. M.; Keri, R. S.; Hugar, M. H. Eur. J. Med. Chem. 2010, 45, 1753-1759.

[23]. Ahmed, S. A.; Hamdy, M. A. -R.; Nadia, M. M.; Mahmoud, A. E. -G. Bioorg. Med. Chem. 2006, 14, 1236-1246.

[24]. Saitoh, M.; Kunitomo, J.; Kimura, E.; Hayase, Y.; Kobayashi, H.; Uchiyama, N.; Kawamoto, T.; Tanaka, T.; Mol, C. D.; Dougan, D. R.; Textor, G. S.; Snell, G. P.; Itoh, F. Bioorg. \& Med. Chem. 2009, 17, $2017-$ 2029.

[25]. Sen Gupta, A. K.; Garg, M.; Chandra, U. J. Indian Chem. Soc. 1979, 56, 1230-1232.

[26]. Chiyomaru, I.; Takita, K.; Ito, H.; Kumiai Chem. Ind. Co. Ltd., Jap. Pat. 1972, 72 07, 549. Chem. Abstr. 1972, 77, 549.

[27]. ONeal, J. B.; Rosen, H.; Russel, P. B.; Adams, A. C.; Blumenthal, A. J. Med. Pharm. Chem. 1962, 5, 617-626.

[28]. Thomas, J. Ger. Pat. 2, 403, 357/1974 Chem. Abstr. 1974, 81, 136153g.

[29]. Yale, H. L.; Losee, K. J. Med. Chem. 1966, 9, 478-483.

[30]. Turner, S.; Reckitt and Colman Products Ltd., Ger. Pat. 1978, 2, 727, 146 Chem. Abstr., 1978, 88, 105357s.

[31]. Hodogaya Chemical Co. Ltd., Jap. Pat. 1980, 8027024 Chem. Abstr., $1980,93,232719 q$

[32]. Hakko Chem. Ind. Co. Ltd., Brit. Pat. 1, 266, 542/1972 Chem. Abstr., 1972, 77, 5474g.

[33]. Singh, H.; Yadav, L. D. S. Agric. Biol. Chem. 1976, 40, 759-764.

[34]. Misato, T.; Ko, K.; Honma, Y.; Konno, K.; Taniyama, E. Inst. Phys. Chem. Res., Jap. Pat. 1977, 772508 Chem. Abstr. 1977, 87, 147054

[35]. Vassiliou, S.; Kosikowska, P.; Grabowiecka, A.; Yiotakis, A.; Kafarski, P.; Berlicki, L. J. Med. Chem. 2010, 53, 5597-5606.

[36]. Vassiliou, S.; Grabowiecka, A.; Kosikowska, P.; Yiotakis, A.; Kafarski, P.; Berlicki, L. J. Med. Chem. 2008, 51, 5736-5744.

[37]. Rauf, A.; Ahmed, F.; Qureshi, A. M.; Aziz-ur-Rehman, K. A.; Qadir, M. I.; Choudhary, M. I.; Chohan, Z. H.; Youssoufi, M. H.; Hadda, T. B. J. Chin. Chem. Soc. 2011, 58, 528-537.

[38]. Muri, E. M. F.; Mishra, H.; Stein, S. M.; Williamson, J. S. Lett. Drug Des. Discov. 2004, 1, 30-34.

[39]. Khan, M. H., Hameed, S.; Yasin, K. A.; Akhtar, T.; Khan, K. M. Monatsh Chem. 2010, 141, 479-484.

[40]. Serwar, M.; Akhtar, T.; Hameed, S.; Khan, K. M. Arkivoc 2009, 7, 210221.

[41]. Amtul, Z.; Rasheed, M.; Choudhary, M. I.; Rosanna, S.; Khan, K. M.; Attaur-Rahman, Biochem. Biophys. Res. Commun. 2004, 319, 1053-1063.

[42]. Weatherburn, M. W. Anal. Chem. 1967, 39, 971-1974.

[43]. Khan, K. M.; Shahzad, S. A.; Rani, M.; Ali, M.; Perveen, S.; Anwar, A.; Voelter, W. Lett. Org. Chem. 2006, 3, 286-288. 